\title{
OMNI-HISTOGRAMS: A NOVEL PARTITIONING APPROACH FOR SIMILARITY SEARCH
}

\author{
Marcos V. N. Bedo \\ Fluminense Northwest Institute/Fluminense Federal University - (INFES/UFF) \\ Av. João Jasbick, S/N - Aeroporto - S. A. Pádua/RJ - Brazil \\ marcosbedo@id.uff.br \\ Rodolfo A. de Oliveira \\ Fluminense Northwest Institute/Fluminense Federal University - (INFES/UFF) \\ Av. João Jasbick, S/N - Aeroporto - S. A. Pádua/RJ - Brazil \\ rodolfooliveira@id.uff.br \\ Daniel S. Kaster \\ Dept. of Comp. Science/State University of Londrina - Brazil - (DC/UEL) \\ Celso Garcia Cid, S/N - C. Universitário - Londrina/PR - Brazil \\ dskaster@uel.br \\ Daniel C. M. de Oliveira \\ Institute of Computing/Fluminense Federal University - (IC/UFF) \\ Gal. Milton Tavares de Souza, S/N - S. Domingos - Niterói/RJ - Brazil \\ danielcmo@ic.uff.br \\ Caetano Traina Jr. \\ Institute of Math. and Comp. Sciences/University of São Paulo - (ICMC/USP) \\ Av. Trab. São Carlense, 400 - Centro - S. Carlos/SP - Brazil \\ caetano@icmc.usp.br
}

\begin{abstract}
Similarity searching supports several computational tasks, such as classification and content-based retrieval. A plethora of indexes has been proposed aiming at enhancing similarity queries, being the Omni-family one of the most versatile. The main strength of Omni methods is they handle the data elements regarding a small set of carefully selected pivots. In this study, we improve the Omni-family and create a new class of indexes called Omni-histograms. Our approach summarizes distance distributions to Omni-pivots in such a way histograms' buckets are also employed for the partitioning of the search space into disjoint regions. The resulting structures boost query executions by using the frequency within each region for limiting both disk accesses and distance calculations. Experiments on real-world datasets showed our approach outperforms existing methods in up to $113 \%$.
\end{abstract}

Keywords: Similarity search; access methods; histograms; $k$-NN queries; pivot selection. 


\section{Como Citar:}

BEDO, M.; OlIVEIRA, R.; KASTER, D.; OliVEIRA, D.; TRAINA JR., C.. OmniHistograms: A novel partitioning approach for similarity search. In: SIMPÓSIO DE PESQUISA OPERACIONAL E LOGÍSTICA DA MARINHA, 19., 2019, Rio de Janeiro, RJ. Anais [...]. Rio de Janeiro: Centro de Análises de Sistemas Navais, 2019.

\section{INTRODUCTION}

Similarity searching is one of the most employed paradigms for the handling and querying of data that are "alike" but not "equal". The paradigm supports a broad variety of computational tasks, such as clustering, classification, and contentbased retrieval [1, 2, 3]. In those tasks, a type of query that is often requested in practice is the $k$-nearest neighbor $(k$-NN) query. Examples of $k$-NN searches include: (Q1) Find the 3 closest beaches to 'Copacabana Beach', and (Q2) Find the 5 paintings of the 'Renaissance period' which are the most similar to 'Mona Lisa'. Notice (Q2) contains a filtering condition on the orthogonal attribute 'Art Period', which limits the candidates to the query answer [4, 5].

Different metric access methods have been proposed to speed up similaritybased queries [6, 7, 8]. Such methods accelerate similarity searches by targeting an optimization criterion, such as the number of disk accesses, the number of distance calculations, or the overhead caused by the querying algorithm [9]. One of the most versatile strategies for improving $k$-NN search is the Omni approach [8], which aims at reducing both distance calculations and disk accesses. It uses a few, but relevant, elements from the dataset as pivots to create new and multidimensional representation for each data element [10]. If an underlying access method is employed for the handling of these new representations, then distance calculations are avoided by following both the triangle inequality distances to the pivots and the pruning rules of the underlying structure. Therefore, the Omni approach can be implemented on top of disk-based indexes, such as B-Tree [11], R-Tree [12], or even Sequential Scan. Notice a new access method is generated whenever the Omni approach is coupled to an existing one, which creates the Omni-family of access methods [8].

Traditionally, statistics derived from distances between data elements are employed for enhancing the execution of similarity searches [13, 14, 15]. Moreover, recent studies indicate distance distributions characterize the effectiveness of metric indexing strategies [13, 16, 17]. Seizing the performance enhancement brought by the Omni approach, this paper employs pivot-based distance histograms for the creation of a new class of access methods, which we call Omni-histograms. They distinguish themselves by their partition constraints, which leads to distinct organizations of the search space and different performances in the execution of $k$-NN queries. In this paper, we focus on five Omni-histogram variants that cover most of the partition strategies, namely Equi-Width, Equi-Depth, V-Optimal, Compact-distance Histogram and Curve-Fitting. The idea behind our structures is the creation of disjoint and pivot-indexed regions, which are also the bucket boundaries for histograms of distance distributions collected from the pivots' perspective. Accordingly, the frequency within each region is available before the query execution and enables the bounding of both disk accesses and distance calculations. 
We performed extensive experiments on real-world datasets for comparing the performance of Omni-histograms and previous Omni-family methods in the task of executing $k$-NN queries with and without orthogonal attributes. The results indicate Omni-histograms achieved significant gains for queries in arbitrary search spaces. Therefore, the contributions of this study are summarized as follows:

1. We introduce the Omni-histogram class of access methods. Omni-histograms organize the search space according to pivot-based distance distributions, and

2. We propose a bounded and incremental $k$-NN search algorithm over Omnihistograms whose parameters are automatically calculated.

The remainder of the paper is organized into four sections. Section 2 provides the background on similarity searching and discusses related work. Section 3 introduces the Omni-histograms, their settings, and algorithms. Section 4 provides an evaluation of Omni-histograms, while Section 5 concludes the paper.

\section{BACKGROUND AND RELATED WORK}

\subsection{Similarity searching}

Similarity searching is the information retrieval process in which the query includes an element of a domain, and the answer is composed of a set of elements of the same domain that are somehow similar to the query instance [18]. Among the several types of similarity queries, two are the most basic, namely the range query and the $k$-nearest neighbor $(k$-NN) query [1, 9]. Let $\mathbb{S}$ be a data domain, $S \subseteq \mathbb{S}$ be a set of elements, and $\delta$ be a metric that holds the properties of symmetry, nonnegativity, and triangle inequality, then the pair $\langle\mathbb{S}, \delta\rangle$ is a metric space in which similarity searches are performed [19]. Given a query element $s_{q}$, a range query $R q$ retrieves all elements of $S$ which are at most a given threshold $\xi \in \mathbb{R}_{+}$from $s_{q}$ such that $R q\left(S, s_{q}, \xi\right)=\left\{s_{i} \in S \mid \delta\left(s_{i}, s_{q}\right) \leq \xi\right\}$. In contrast, $k$-NN queries return a quantity $k$ of elements whose distance to the query element $s_{q}$ are the smallest. Therefore, a $k$-NN query is a variation of the $R q$ query, i.e., a $R q$ with a set radius $\xi$ such that $|R q|=k$ [14]. The pair $\left\langle s_{q}, \xi\right\rangle$ defines a closed query ball in the search space, which covers more or fewer elements according to $\xi$. Therefore, small values of $\xi$ may lead to empty result sets, whereas if $\xi$ is indiscriminately increased, all elements of $S$ can be returned [2, 20]. Since $k$-NN queries enable to control the result cardinality, reducing their execution time is the focus of our investigation.

\subsection{The Omni-family of access methods}

Pivot-table strategies [9, 10] rely on precomputing and storing distances $\delta\left(s_{i}, p\right)$ of the elements $s_{i} \in S$ to a set of pivots $p \in \mathcal{P}$. Therefore, given a range query $R q\left(S, s_{q}, \xi\right)$, the triangle inequality property of metric distance functions ensures elements $s_{j} \in S$ outside the query ball $\left\langle s_{q}, \xi\right\rangle$ comply with pruning rule $\mid \delta\left(s_{j}, p\right)-$ $\delta\left(p, s_{q}\right) \mid>\xi$ for at least one pivot $p \in \mathcal{P}$ [18]. The Omni approach [8] combines such a pruning rule with the clustering of precomputed distances to reduce both distance 
calculations and random disk accesses by means of the querying algorithm. The approach clusters the precomputed distances by means of a broad set of underlying access methods so that incremental $k$-NN searches [21] can be executed. Accordingly, both the triangle inequality distance to the pivots and the pruning rules of the underlying access method are used for avoiding distance calculations. Omni-pivots $p \in \mathcal{P} \subseteq S$ are fetched in linear time by the Omni Hull-Foci algorithm, and the number of pivots is calculated as the dataset fractal dimension $(\lceil\mathcal{D}\rceil)[8]$. Every element $s_{i} \in S$ is mapped into an Omni-coordinate through $\mathcal{P}$, as in Definition 1 . Figure 1 provides an example of such mapping for (a) one, and (b) two pivots.

Definition 1 (Omni-coordinate). Given an ordered set of pivots $\mathcal{P}$ and an element $s_{i} \in \mathbb{S}$, the Omni-coordinate $O\left(s_{i}\right)$ of $s_{i}$ is the set of distances from $s_{i}$ to each pivot $p \in \mathcal{P}$ such that $O\left(s_{i}\right)=\left\{\delta\left(s_{i}, p_{1}\right), \ldots, \delta\left(s_{i}, p_{|\mathcal{P}|}\right)\right\}$. The set of Omni-coordinates of all elements $s_{i}$ in a dataset $S$ is denoted by $O_{S}$.

As for Omni implementation, the approach relies on an abstraction layer that includes $S, \mathcal{P}, O_{S}$ and a map between $S$ and $O_{S}$. Any existing access method can become part of the Omni-family by extending the abstraction layer and providing a partition to $O_{S}$.

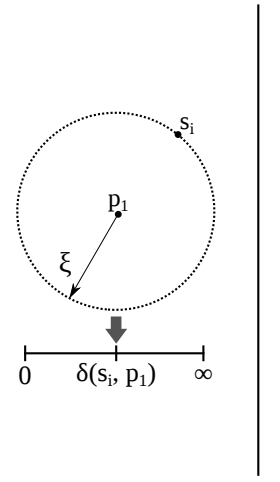

(a)

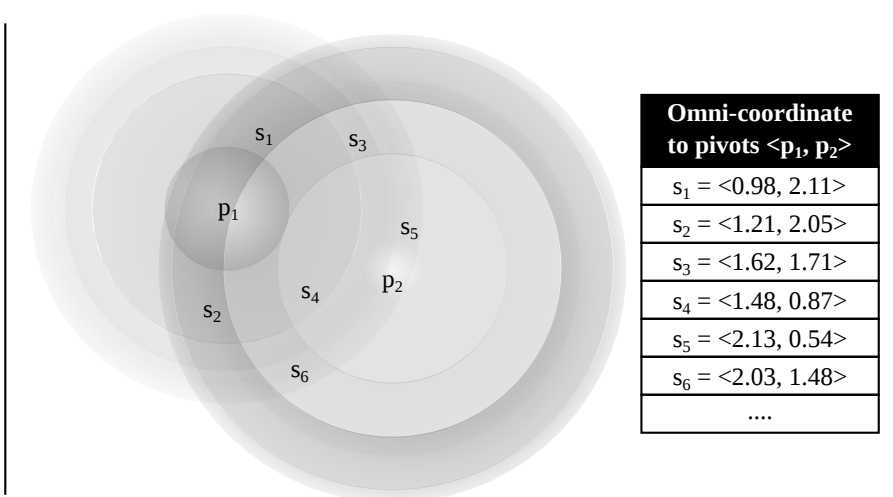

(b)

Figure 1: Mapping of a metric space into a multidimensional space according to the $L_{2}$ distance. (a) Map of distances for a single pivot $p_{1}$ to a one-dimensional space. (b) Map and distance distribution for 100 random points and two pivots.

\subsection{Solving of $k$-NN Queries by Best-First Search}

Unlike range queries, $k$-NN queries do not have a radius defined beforehand and, therefore, the access method cannot draw a query ball for limiting the search space [14, 15]. Alternatively, clustered access methods' algorithms employ a branchand-bound strategy for the pruning of regions during the query [6]. Such a strategy initially sets the radius to a maximum $(\xi=\infty)$ and dynamically reduces it until the $k$-nearest neighbors have been found. For a faster radius reduction, regions are evaluated in order of proximity to the query element, which results in a procedure known as best-first search (bf-kNN).

An optimization of bf-kNN is the estimation of the initial radius $\xi^{\prime}$, which delimits a search region, and reduces the number of disk accesses and distance 


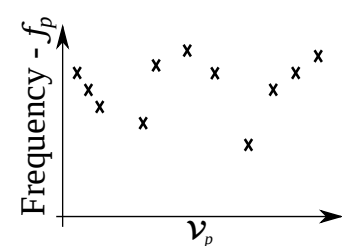

(a)

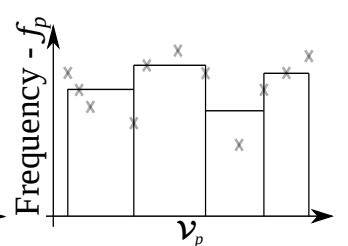

(b)

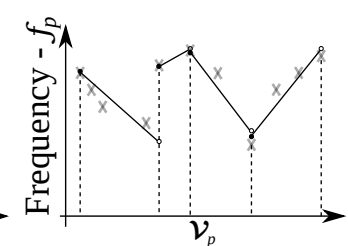

(c)

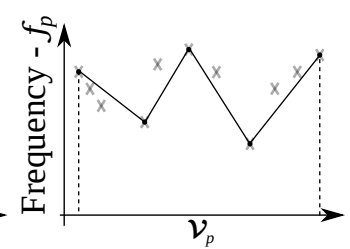

(d)

Figure 2: Histograms of $\mathcal{T}_{p}$ with a fixed number of buckets and distinct partition constraints. (a) Original pivot-based distribution. (b) V-Optimal histogram. (c) Curve-Fitting histogram. (d) Compact-distance histogram.

calculations. In this case, estimated radius $\xi^{\prime}$ and $s_{q}$ define the query ball, and the access method executes a combination of range and bf-kNN to return the $k$ closest elements [14] - A procedure we call limited $b f-k N N$. Radius estimation depends on metric statistics and requires the gathering of distance distributions [14, 19]. Such statistics can be calculated following viewpoints, in the form of pivot-based distance distributions, which are formally given by Definition 2 .

Definition 2 (Pivot-based distance distribution $-\mathcal{T}_{p}$ ). Given a dataset $S$, a metric $\delta$, and a pivot $p \in \mathcal{P}, \mathcal{T}_{p}$ captures the distance from each $s_{i} \in S$ to $p$. Distance value set $\mathcal{V}_{p}$ contains the distinct and sorted values of $\delta\left(s_{i}, p\right)$, i.e., $\mathcal{V}_{p}=\left\{v_{p}(j): 1 \leq j<\right.$ $\left.m_{p}, m_{p} \leq|S|\right\}$, where $v_{p}\left(m_{p}\right)$ is the largest distance between any $s_{i}$ to $p$. Frequency $f_{p}(j)$ is the number of elements of $S$ whose distance $\delta\left(s_{i}, p\right)=v_{p}(j)$. Therefore, $\mathcal{T}_{p}$ is the ordered set of pairs $\mathcal{T}_{p}=\left\{\left\langle v_{p}(1), f_{p}(1)\right\rangle, \ldots,\left\langle v_{p}\left(m_{p}\right), f_{p}\left(m_{p}\right)\right\rangle\right\}$, and $\mathcal{T}_{p}^{+}$is the extension of $\mathcal{T}_{p}$ to $\mathbb{R}$ by setting 0 as the frequency of any $v_{p} \in \mathbb{R}_{+} \backslash \mathcal{V}_{p}$.

Histograms [22] can be employed for the summarization of pivot-based distance distributions. In this case, the histogram sort and source parameters are $\mathcal{V}_{p}$ and the set of frequencies, respectively. Distances within each bucket are uniformly distributed, but frequencies are approximate according to a user-posed histogram partition constraint. Hence, a histogram $H_{p}=\left\{b_{1}, \cdots, b_{\beta}\right\}$ partitions either $\mathcal{T}_{p}$ or $\mathcal{T}_{p}^{+}$into $\beta$ mutually disjoint buckets. Each bucket $b_{i}$ covers a range of the value set such that $0 \leq \operatorname{low}\left(b_{i}\right)<u p\left(b_{i}\right) \leq \operatorname{low}\left(b_{i+1}\right)<u p\left(b_{i+1}\right)<\infty$, where low $\left(b_{i}\right)$, up $\left(b_{i}\right) \in \mathcal{V}_{p}$. For the partitioning of $\mathcal{T}_{p}^{+}$an extra bucket $b_{\beta+1}$ is employed in the interval $\left(u p\left(b_{\beta}\right), \infty\right)$ with frequency 0. Examples of classical partition constraints include the Equi-Width and Equi-Depth histograms [13, 22, 23] constructed with uniformly spaced buckets regarding $v_{p}$ (distance value) and $f_{p}$ (frequency), respectively. More complex constraints involve the optimization of an objective function in the partitioning of the source parameter. For instance, V-Optimal histograms partition $\mathcal{T}_{p}$ by minimizing the variance of the frequencies within each bucket. Curve-Fitting histograms [23] improve V-Optimal by approximating the frequencies through a set of polynomial functions, while Compact-distance histograms [13] simplify Curve-Fitting histograms by approximating $\mathcal{T}_{p}$ as a continuous piecewise linear function. Figure 2 shows examples of histograms for $\mathcal{T}_{p}$ following the former three partition constraints, which generate different approximation errors.

\subsection{Limited Best-First Search vs. Incremental Search}

Another approach for the execution of $k$-NN queries is the incremental search [21], a procedure known as inc-kNN. Such a strategy limits the number of 
distance calculations based on an optimality principle. The idea is to incrementally retrieve the closest element to the query instance by using two priority queues. The first queue sorts the partitions to be evaluated, while the second one sorts the elements of the already examined regions, which include the next potential nearest neighbor. Elements in the second priority queue are sorted by their distances to the query instance, while the partitions in the first queue are sorted by the minimum and maximum distances between their boundaries and the query element. The decision to select the next nearest neighbor is made upon the evaluation of both queues. If the top of the second queue is closer to the query instance than the minimum distance of the region on top of the first queue, then the first element of the second queue is the next nearest neighbor. Otherwise, the partition of the first queue must be loaded from disk and its elements be inserted into the second priority queue.

One advantage of inc-kNN is it enables solving queries with orthogonal attributes, as in the query example Q2 about the nearest Renaissance paintings most similar to 'Mona Lisa', a feat that limited bf-kNN is unable to accomplish. However, inc-kNN may require an execution time greater than limited bf-kNN in other cases [14, 15]. It happens because inc-kNN must handle two expensive priority queues (especially the second one) for the solving of $k$-NN queries, which generates an overhead in both memory and processing. Moreover, inc-kNN does not define a query ball and, consequently, it may not benefit from elevator-based disk scheduling that could improve the $k$-NN search performance.

In this study, we combine the best of both inc-kNN and limited bf-kNN approaches in the form of a bounded and incremental $k$-NN algorithm on top of Omni-histograms. Our approach enables the definition of a query ball and the sorting of partitions and elements into priority queues, which limits on-demand disk accesses and leads to a reduction in the number of distance calculations.

\section{THE OMNI-HISTOGRAMS}

This section proposes the Omni-histograms, a new and robust class of metric access methods that extends the abstraction layer of the Omni-family. The addition of histograms into the Omni approach enables both the partitioning of Omni-coordinates and $k$-NN search optimization. In particular, Omni-histograms divide the search space into disjoint regions by using the Omni-pivots, whereas the final number of regions depends on the number of buckets for each pivot-based histogram. The following parameters define a specific instance of an Omni-histogram: (i) the number of pivots $|\mathcal{P}|$, (ii) the maximum number of buckets $\beta$, and (iii) a histogram partition constraint $\mathcal{R}$. The overall idea is to take advantage of the Omni abstraction layer for the gathering of extended pivot-based distributions so that they can be clustered according to the histogram partition constraint.

Therefore, an Omni-histogram can be seen as the set of non-independent pivot-based distance histograms derived from the set of Omni pivots. Formally, an Omni-histogram $\mathcal{H}$ is defined as a set of pairs $\mathcal{H}=\left\{\left\langle p, H_{p}\right\rangle, p \in \mathcal{P}\right\}$, where $H_{p}$ is the histogram partitioning of $\mathcal{T}_{p}^{+}$. Figure 3 shows an example of a $2 \mathrm{D}$ dataset with geographical coordinates of Brazilian cities and its partitioning by two distinct 


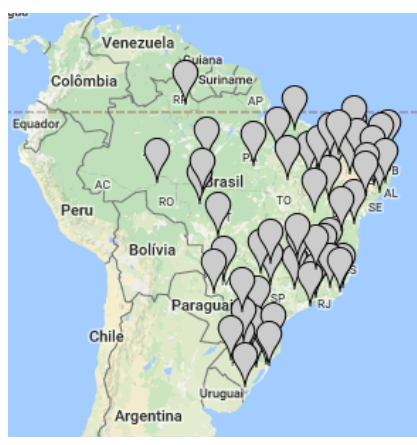

(a)

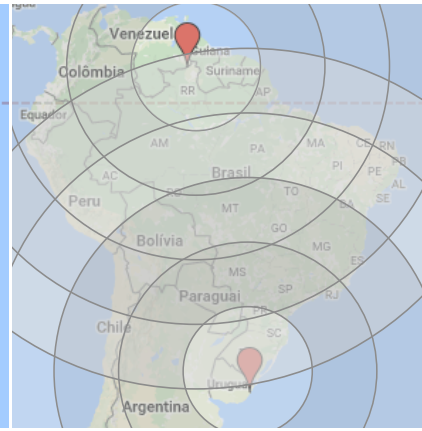

(b)

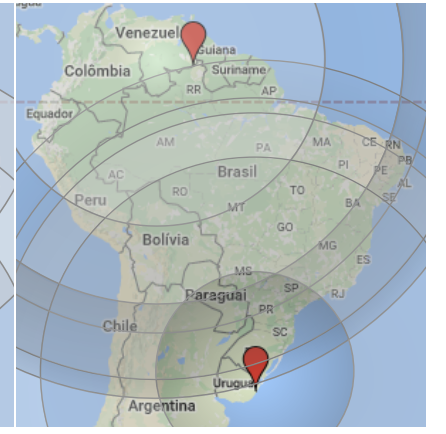

(c)

Figure 3: A dataset with geographical coordinates where most cities are closer to the coast. Histograms are constructed for two pivots, $\beta=5$ buckets, and $L_{2}$ distance. The darker regions are the denser ones. (a) A sample of 60 medoids. (b) Equi-Width Omni-histogram regions. (c) Compact-distance Omni-histogram regions.

Omni-histograms. Figure 3(a) presents a sample of the spatial distribution of 60 medoids found by the $k$-medoids clustering algorithm, while Figures 3(b) and (c) show the Equi-Width Omni-histogram and Compact-distance Omni-histogram built for the same dataset with regards to $L_{2}$ distance.

An Omni-bucket $b^{*}$ covers a hyper region in the search space defined by a set of buckets from distinct pivot-based histograms in $\mathcal{H}$. The boundaries of an Omnibucket $b^{*}$ are the limits of buckets $b_{i} \in H_{p}$ related to $b^{*}$ and $\mathcal{H}$ by pivots $p \in \mathcal{P}$. Each element of $S$ falls into only one Omni-bucket calculated by the distance between the element and $\mathcal{P}$. The frequency of each Omni-bucket is the number of elements which lie inside the corresponding hyper region. We call Omni bucket-coordinate the mapping between an element $s_{j} \in \mathbb{S}$ and its Omni-bucket as in Definition 3.

Definition 3 (Omni bucket-coordinate). Given an Omni-histogram $\mathcal{H}$ and an element $s_{j} \in \mathbb{S}$, the Omni bucket-coordinate $B\left(s_{j}\right)$ of $s_{j}$ addresses the Omni-bucket $b^{*}$ of $\mathcal{H}$ whose limits include $s_{j}$. Therefore, $B\left(s_{j}\right)=\left\{\left\langle p, b_{i}\right\rangle \mid \forall\left\langle p, H_{p}\right\rangle \in \mathcal{H}, b_{i} \in\right.$ $H_{p} ;$ low $\left.\left(b_{i}\right) \leq \delta\left(s_{j}, p\right)<u p\left(b_{i}\right)\right\}$. If the order for pivot set $\mathcal{P}$ is fixed, then $B\left(s_{j}\right)$ can be written as $B\left(s_{j}\right)=\left\{b_{i 1}, \ldots, b_{i|\mathcal{P}|}\right\}$. The set of Omni bucket-coordinates regarding all elements of $S$ is denoted $B_{S}$.

Omni-histograms organize the search space into three levels. The first level includes the Omni-buckets, $B_{S}$ and a map between $O_{S}$ and $B_{S}\left(\mathcal{M}\left(O_{S}, B_{S}\right)\right.$, for short). The second level includes the Omni-coordinates $O_{S}$ and a map between $S$ and $O_{S}\left(\mathcal{M}\left(S, O_{S}\right)\right.$, for short $)$. Finally, the third level includes all the elements of $S$ and their mapping to $O_{S}$ and $B_{S}\left(\mathcal{M}\left(O_{S}, B_{S}\right)\right.$, for short). Figure 4 shows an example of the relationship between Omni-bucket coordinates and Omni-coordinates.

\subsection{Generalized Omni-Histograms}

The frequency of an Omni-bucket $b^{*}$ can be expressed as the distribution of elements within $b^{*}$ regarding any orthogonal attribute $A$. In this case, the frequency of $b^{*}$ itself is a histogram on $A$ regarding the data distribution of elements inside the hyper region delimited by $b^{*}$. We generalize Omni-histograms by expressing the 


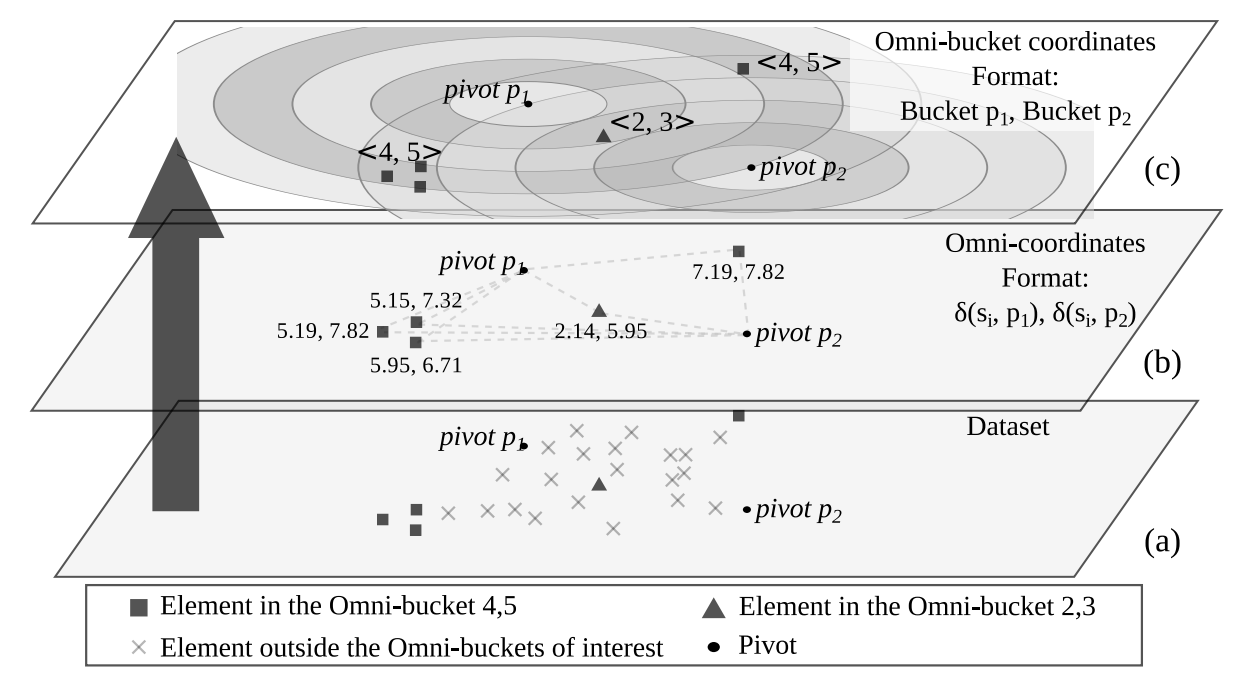

Figure 4: Omni-histogram partitions for Omni-buckets $\langle 2,3\rangle$ and $\langle 4,5\rangle$ regarding pivots $p_{1}$ and $p_{2}$. (a) Data elements. (b) Instances are mapped according to its Omni-coordinates that define two distributions: from $p_{1}$, and $p_{2}$. (c) Distance distributions are partitioned into Omni-buckets that reference their elements.

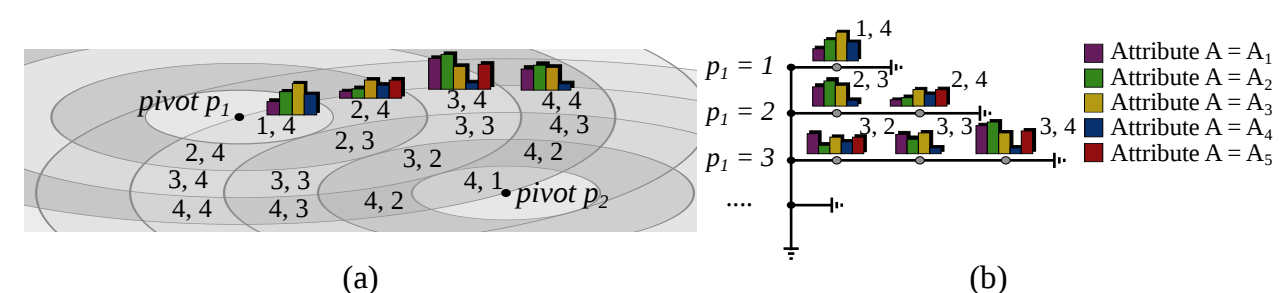

(a)

(b)

Figure 5: Structure of a generalized Omni-histogram. (a) Equi-Width Omni-histogram. (b) Adjacency list for Equi-Width histograms on a orthogonal attribute, which are the frequencies of Omni-buckets.

frequency of Omni-buckets as separated histograms, whereas the histogram build for the orthogonal attribute may comply with a partition constraint distinct from that of $\mathcal{H}$. Figure 5 shows an example of a generalized Omni-histogram in which the hyper regions are enumerated by their Omni bucket-coordinates, and the frequency of each Omni-bucket is an Equi-Width histogram on an orthogonal attribute $A$.

Algorithm 1 constructs a generalized Omni-histogram by using pivot-based distance distributions on attribute $S$ and data distributions on attribute $A$. The algorithm maps the elements of $S$ into the Omni-buckets by calculating their coordinates. Next, it builds a separated histogram $H_{A}$ for each Omni-bucket $b^{*}$ of $\mathcal{H}$ regarding the attribute $A$ by taking into account the elements covered by $b^{*}$. Finally, histograms $H_{A}$ are set as the Omni-buckets' frequencies.

\subsection{Solving $k$-NN Queries with Omni-Histograms}

The $k$-NN search in Omni-histograms is optimized regarding two aspects: (i) disk accesses are calculated beforehand as in range queries, and (ii) distance calculations are minimized through incremental processing. Omni-histograms estimate 


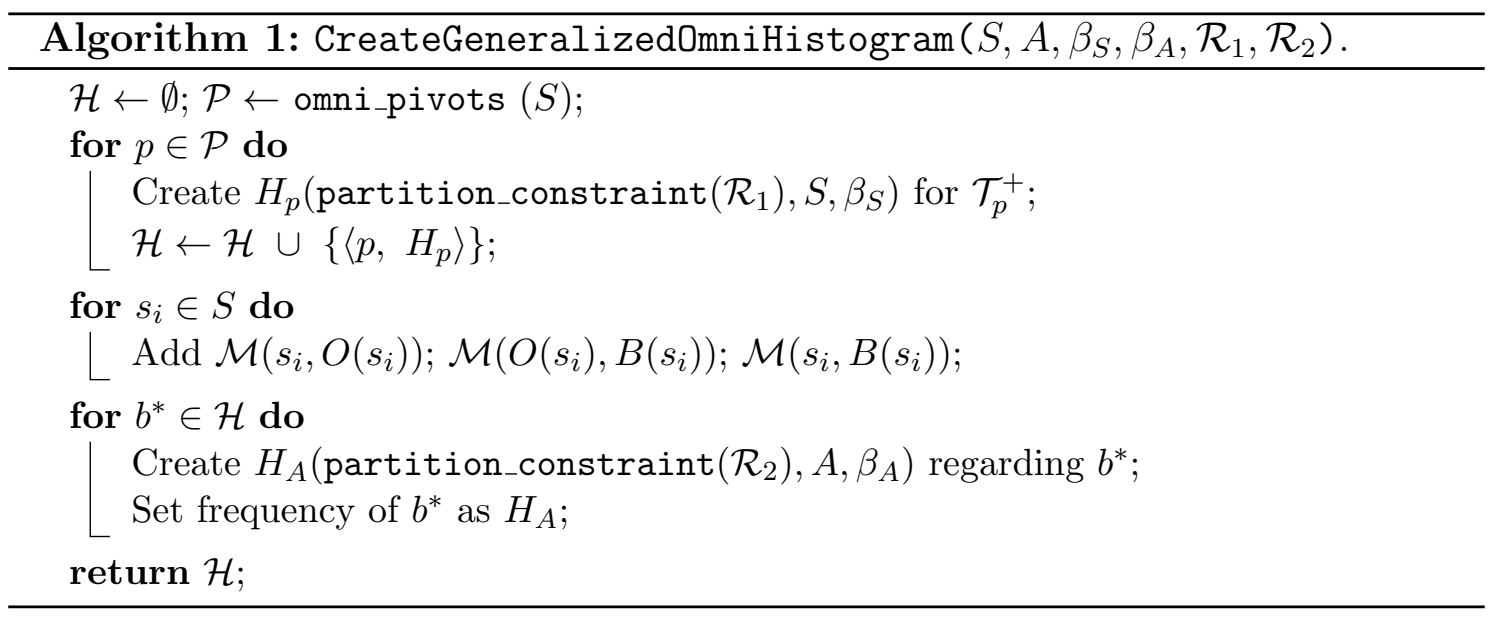

the minimum radius that defines a query ball in which it is ensured at least $k$ elements can be found for the $k$-NN query, whereas only Omni-buckets that intercept the query ball are load into main memory for evaluation. The minimum number of elements within an Omni-bucket is calculated by using the frequency within the partition and a possible query-imposed filter on the orthogonal attribute. Routine numElements () is implemented for such a calculation and returns the accumulated area within the histogram on the orthogonal attribute whose data values comply with query criteria.

Omni-histograms minimize the number of distance calculations for the nondiscarded buckets by using two baseline functions: maxdist() and mindist(). Func-

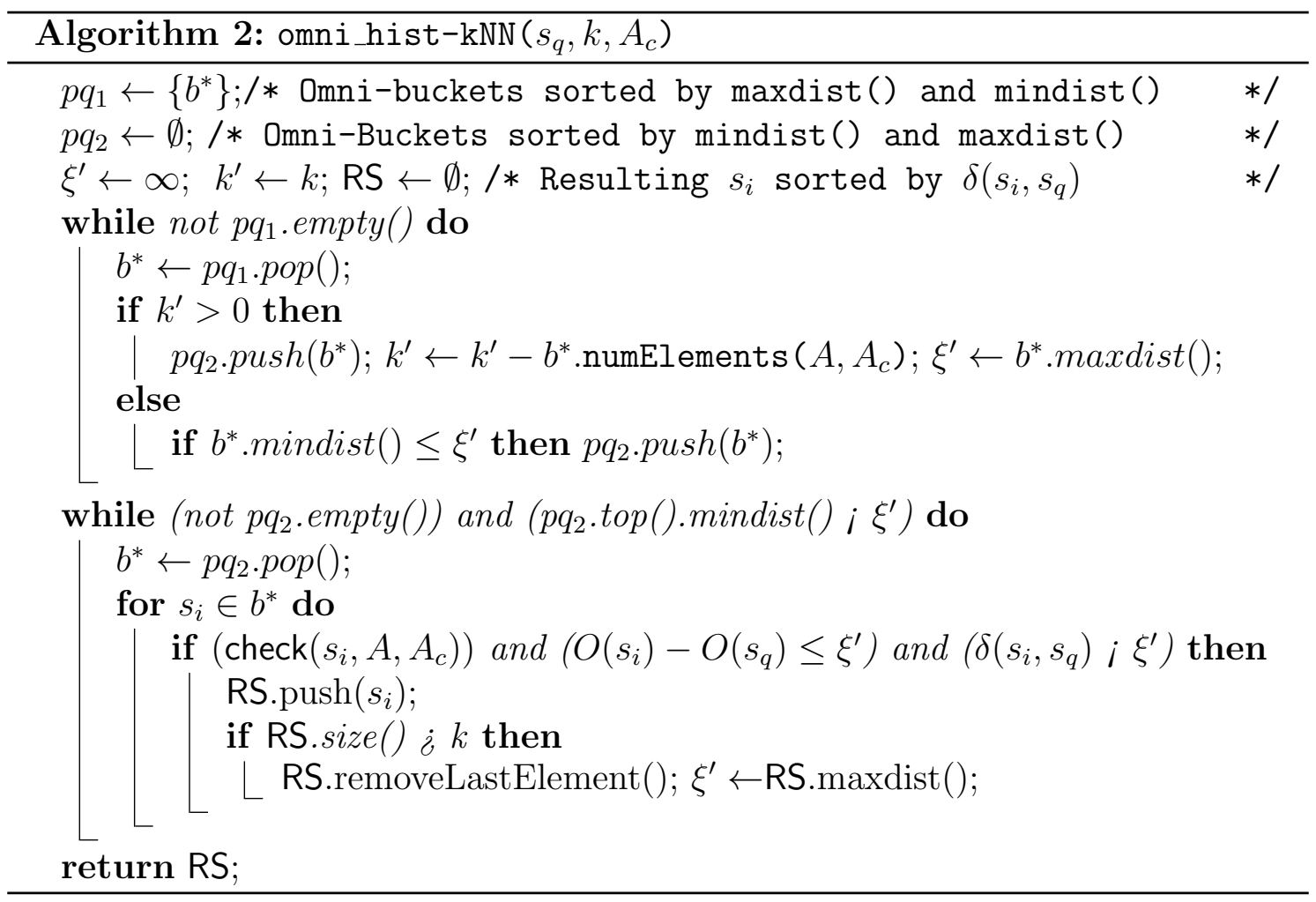


tion mindist() of the query element $s_{q}$ to an Omni-bucket $b^{*} \in \mathcal{H}$ is the minimum distance between $s_{q}$ and a boundary of $b^{*}$. It is calculated as $\min \left(\mid \operatorname{low}\left(b_{i}\right)-\right.$ $\left.\delta\left(s_{q}, p\right)|| u p,\left(b_{i}\right)-\delta\left(s_{q}, p\right) \mid\right) \forall p \in \mathcal{P}$, where $b_{i}$ is the bucket of $H_{p}$ that defines $b^{*}$. On the other hand, maxdist () of $b^{*} \in \mathcal{H}$ is calculated as $\max \left(u p\left(b_{i}\right)+\delta\left(s_{q}, p\right)\right) \forall p \in \mathcal{P}$. Therefore, the combination of functions mindist() and maxdist() with routine numElements () enables the cutting of partitions that do not include any candidate element to the query answer. In particular, numElements() defines the query ball, whereas mindist() enables the incremental evaluations of Omni-buckets covered by the query ball that include the $k$-nearest elements to $s_{q}$.

Algorithm 2 describes a $k$-NN search on Omni-histograms for a query element $s_{q}$ and a filter $A_{c}$ on the orthogonal attribute $A$. First, it limits the number of disk accesses by selecting and sorting the candidate Omni-buckets that intercept the query ball and proceeds with the goal of delaying the distance calculations as much as possible. Next, Algorithm 2 starts a loop on $p q_{2}$ that runs until $k$-nearest neighbors are found or $p q_{2}$ becomes empty. The closest bucket $b^{*}$ within $p q_{2}$ is picked for evaluation, and their elements $s_{i}$ are verified through the filtering criteria on the orthogonal attribute by boolean routine $\operatorname{check}\left(s_{i}, A, A_{c}\right)$. If the criteria are satisfied, the algorithm applies the triangle inequality rule $O\left(s_{i}\right)-O\left(s_{q}\right) \leq \xi^{\prime}$, which verifies if at least one of the precomputed distances satisfies $\left|\delta\left(s_{i}, p\right)-\delta\left(p, s_{q}\right)\right| \leq \xi^{\prime}$.

Therefore, the distance between $s_{i}$ and $s_{q}$ is calculated only when the criteria on the orthogonal attribute are satisfied, and no pruning is performed in the Omnicoordinates. In such a case, $s_{i}$ is inserted into the result set $R S$, a priority queue sorted by distance $\delta\left(s_{i}, s_{q}\right)$. If $s_{i}$ is selected for insertion into $R S$ and the result set has already $k$ elements, the algorithm pushes $s_{i}$ into $R S$, removes the last element of the priority queue, and updates the pruning radius $\xi^{\prime}$. Figure 6 illustrates the running of Algorithm 2 for query example (Q2) that retrieves the five Renaissance paintings which are the most similar to 'Mona Lisa'.

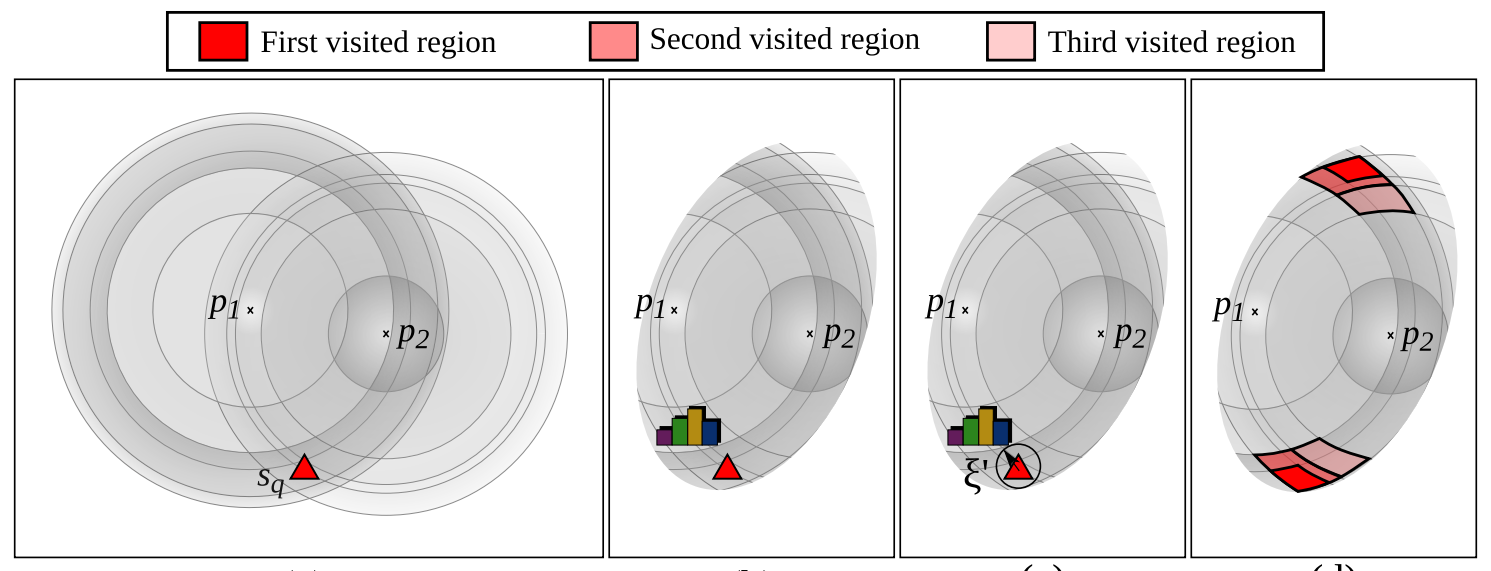

(a)

(b)

(c)

(d)

Figure 6: $k$-NN search example for query element $s_{q}$, filter $A_{c}$, and distance $L_{2}$. (a) Location of $s_{q}$ Omni-bucket coordinate. (b) Histograms on the orthogonal attribute define the query radius $\xi^{\prime}$. (c) Buckets intercepting the query ball are marked for evaluation. (d) Closest regions are visited first. 
We assume an Compact-distance Omni-histogram was constructed for partitioning paintings, whereas the frequencies of each Omni-bucket were described by Equi-Width histograms on orthogonal attribute Art Period. Accordingly, the first step of Algorithm 2 is locating the Omni-bucket corresponding to the 'Mona Lisa' painting so that all remaining Omni-buckets are sorted to the query element by functions mindist() and, then, maxdist() (Figure 6(a)). Next, Equi-Width histograms on the orthogonal attribute are used for calculating the number of elements within every Omni-bucket that satisfy filtering condition Art Period = 'Renaissance' (Figure 6(b)). Accordingly, the sorted list of Omni-buckets is traversed until the accumulated number $k^{\prime}$ of Renaissance elements of visited Omni-buckets become equals or greater than five. At this point, a query ball is defined by using 'Mona Lisa' and a radius $\xi^{\prime}$ corresponding to the maxdist() of the last examined Omnibucket, and the inspection of the sorted list of partitions stops (Figure 6(c)).

The query ball performs the first pruning of Algorithm 2 so that only the Omni-buckets whose mindist() are not greater than $\xi^{\prime}$ are orderly loaded into main memory. One region is evaluated at a time according to its position in the priority queue (Figure 6(d)). For each inspection, the elements within the Omni-bucket are first evaluated by their Omni-coordinates and the filtering condition, which avoids unnecessary distance calculations. When the first candidate set of $k$-nearest neighbors sorted by distance to the query element is built, the query radius $\xi^{\prime}$ is adjusted, and the Omni-buckets of the priority queue in main memory becomes prunable by mindist() once again. If the distance of the instance on top of the candidate result set to the query element is lower than mindist() of the Omnibucket on top of the priority queue, then the instance on top of candidate set can be safely returned as the next nearest neighbor. Accordingly, Algorithm 2 may either incrementally return the nearest neighbor or retrieve the entire set of $k$-nearest neighbors as a single final result.

\section{EXPERIMENTS}

This section reports on three experiments over six real-world datasets, namely CITIES $S^{1}$, BIKE 2 , COLORS ${ }^{3}$, CANVAS $S^{4}$, BANK $^{7}$, and YEAST ${ }^{7}$. The first experiment compares distinct histogram partition constraints for the identification of the most suitable settings of Omni-histograms. The second experiment aims at comparing Omnihistograms to access methods Omni-Sequential, Omni R-Tree and Sequential Scan regarding the execution of $k$-NN queries without orthogonal attributes. Finally, the last evaluation compares the same access methods in the solving of $k$-NN queries with filtering criteria. Omni-Sequential, Omni R-Tree, and Sequential Scan run inc-kNN algorithm, while Omni-histograms run omni_hist-kNN routine.

Table 1 summarizes the datasets characteristics and parameters employed in the trials. All comparisons were performed according to a 10-fold cross validation procedure (90\% of data for indexing and 10\% of data for querying, cycling) regarding

\footnotetext{
${ }^{1}$ Available at: ibge.gov.br

${ }^{2}$ Available at: archive.ics.uci.edu/ml/datasets.html

${ }^{3}$ Available at: sisap.org/metricspaceslibrary.html

${ }^{4}$ Available at: commons .wikimedia.org/wiki/Category:Paintings
} 
accumulated query execution time. The experiments were executed in a computer with Intel ${ }^{\circledR}$ Core $^{\mathrm{TM}}$ i7 $2.67 \mathrm{GHz}, 6 \mathrm{~GB}$ of RAM and HDD SATA III 7200 RPM.

Table 1: Datasets and parameters. $A$ is the orthogonal attribute. $|S|$, Dim., $\lceil\mathcal{D}\rceil$ are the set cardinality, dimensionality, and fractal dimension, respectively.

\begin{tabular}{|l|l|c|c|c|c|l|}
\hline \hline Name & $A$ & $|S|$ & Dim. & $\lceil\mathcal{D}\rceil$ & $\delta$ & Description \\
\hline \hline CITIES & $n / a$ & 5,507 & 2 & 2 & $L_{2}$ & $\begin{array}{l}\text { Geographical coordinates of } \\
5507 \text { Brazilian cities. }\end{array}$ \\
\hline BIKE & $n / a$ & 17,379 & 7 & 2 & $L_{\infty}$ & UCI dataset of bike roads. \\
\hline COLORS & $n / a$ & 112,682 & 112 & 3 & $L_{1}$ & $\begin{array}{l}\text { SISAP dataset of features ex- } \\
\text { tracted from color images. }\end{array}$ \\
\hline \hline BANK & Type & 1,372 & 4 & 2 & $L_{\infty}$ & $\begin{array}{l}\text { UCI dataset of features from } \\
\text { bank notes. }\end{array}$ \\
\hline YEAST & Location & 1,484 & 8 & 4 & $L_{2}$ & $\begin{array}{l}\text { UCI dataset of cellular loca- } \\
\text { tion of proteins. }\end{array}$ \\
\hline CANVAS & $\begin{array}{l}\text { Art Pe- } \\
\text { riod }\end{array}$ & 3,879 & 16 & 4 & $L_{1}$ & $\begin{array}{l}\text { Features extracted from Wiki- } \\
\text { media photos of paintings. }\end{array}$ \\
\hline
\end{tabular}

\subsection{Comparison of Omni-Histograms Settings}

We selected the Omni-pivots according to the Omni Hull-Foci algorithm by setting $|\mathcal{P}|=\lceil\mathcal{D}\rceil$. The constraint number of buckets $\beta$ was chosen following $\mathcal{P}$ so that all Omni-histograms fit in less than $0.0001 \%$ of available memory. In particular, we set five buckets for datasets CITIES, BIKE and BANK, three buckets for dataset YEAST, and two buckets for datasets COLORS and CANVAS. We experimented on five distinct partition constraints that generated five different Omni-histograms,

(a) CITIES

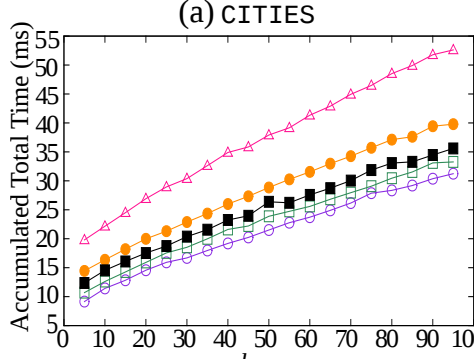

(d) YEAST

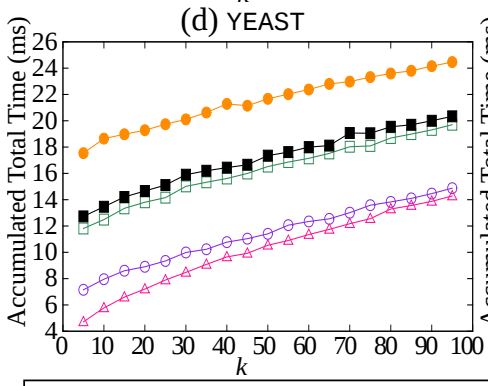
$\square$ Compact-distance Omni-histogram $\rightarrow$-Curve-fitting Omni-histogram

(b) BANK

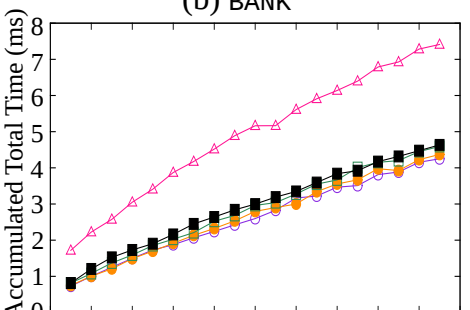

(e) CANVAS

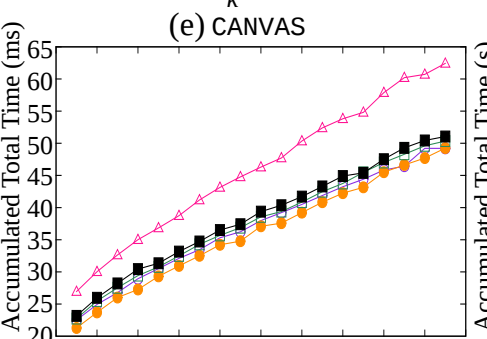

(c) BIKE

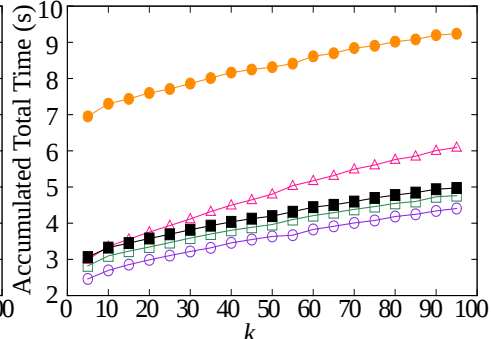

(f) COLORS

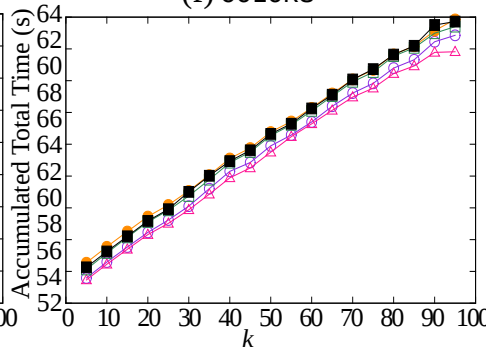

O-Equi-Width Omni-histogram - Equi-Depth Omni-histogram $\triangle$ V-Optimal Omni-histogram

Figure 7: Comparison of distinct Omni-histograms in the execution of $k$-NN queries. 
namely Equi-Width Omni-histogram, Equi-Depth Omni-histogram, V-Optimal Omni-histogram, Compact-distance Omni-histogram, and Curve-Fitting Omni-histogram. Figure 7 shows the overall comparison of the average time required by each Omni-histogram to execute $k$-NN queries without orthogonal attributes.

Although V-Optimal Omni-histogram was the fastest method regarding datasets YEAST and COLORS, it was also one of the slowest on the remaining of experimented datasets. Equi-Depth Omni-histogram followed a similar behavior, i.e., it was the fastest at solving $k$-NN queries on CANVAS, but showed poor performance on other datasets. On the other hand, Equi-Width Omni-histogram and Compact-distance Omni-histogram showed the most stable behavior, as they consistently achieved one of the top-3 performance regardless of the evaluated dataset. Equi-Width Omni-histogram, specifically, achieved the highest performance on datasets CITIES and BIKE. Therefore, we selected both Equi-Width Omni-histogram and Compact-distance Omni-histogram for the comparison of Omni-histograms to other Omni-family access methods.

\subsection{Omni-Histograms vs. Other Members of Omni-Family}

We compared Omni-histograms to access methods Omni-Sequential, Omni R-Tree, and Sequential Scan. Omni-Sequential is the general purpose method of the Omni-family, i.e., it is implemented on top of Sequential Scan, whereas Omni R-Tree employs the R-Tree for the indexing of Omni-coordinates. Figure 8 shows the comparison between the Omni-histograms and previous Omni methods in the execution of $k$-NN queries without orthogonal attributes. Equi-Width Omni-histogram outperformed Omni-Sequential in up to $113 \%$ and $41 \%$ on datasets CITIES and BIKE, respectively. Compact-distance Omni-histogram outperformed OmniSequential in up to $83 \%$ and $30 \%$ in the same scenario.

Moreover, Equi-Width Omni-histogram and Compact-distance Omni-histogram outperformed Omni-Sequential for $k \leq 45$ in dataset COLORS (112 dimensions). In particular, Equi-Width Omni-histogram was up to $5.4 \%$ faster than Omni-Sequential on dataset COLORS, while Compact-distance Omni-histogram was

(a) CITIES

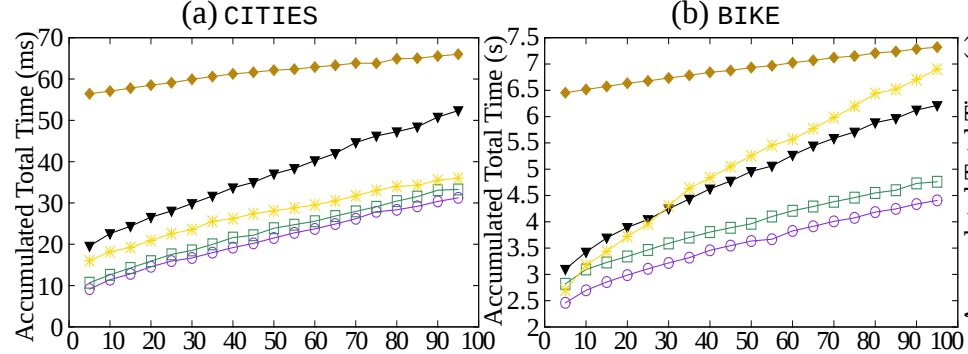

(c) COLORS

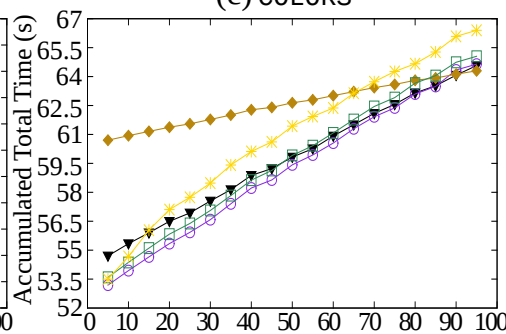

- Equi-Width Omni-histogram $\square$-Compact-distance Omni-histogram $\rightarrow$ Sequential Scan $\rightarrow$ omni-Sequential * Omni R-tree

Figure 8: Comparison of Omni-histograms to Omni R-Tree, Omni-Sequential, and Sequential Scan regarding $k$-NN query execution time. 
up to $3.7 \%$ faster in the same dataset. Both Omni-histograms outperformed Omni $\mathrm{R}$-Tree in the majority of $k$ values.

We highlight Equi-Width Omni-histogram was up to 500\%, $160 \%$ and $16.1 \%$ faster than baseline Sequential Scan on datasets CITIES, BIKE, and COLORS, respectively. Likewise, Compact-distance Omni-histogram also outperformed Sequential Scan in up to $443 \%, 131 \%$ and $12.4 \%$ for the same datasets.

\section{3. $k$-NN searching with Filtering Criteria}

The last experiment provides a comparison between Omni-histograms and their competitors in the task of answering $k$-NN queries with orthogonal attributes. Each query was in the form of "Find the $k$-nearest elements to $s_{q}$, where attribute $A$ equals to $A_{c}$ ", being the orthogonal attribute $A$ of each dataset described in Table 1. Accordingly, we set $A=A_{c}$ as in Type='Class 0' (55.5\% of elements), Location='mitochondrial' (16.4\% of elements), and Art Period='Renaissance' (20\% of elements) for datasets BANK, YEAST, and CANVAS, respectively.

Figure 9 shows the comparison between the access methods regarding their execution time. Equi-Width Omni-histogram outperformed Omni-Sequential by $30 \%$, on average, for every dataset, while Compact-distance Omni-histogram also outperformed Omni-Sequential by 24.3\%, on average. Both Equi-Width Omni-histogram and Compact-distance Omni-histogram outperformed Omni R-Tree for every value of $k$. Equi-Width Omni-histogram also outperformed baseline Sequential Scan in up to $456 \%, 186 \%$ and $147 \%$ for datasets BANK, YEAST and CANVAS. Likewise, Compact-distance Omni-histogram was up to $455 \%, 164 \%$ and $125 \%$ faster than Sequential Scan when executing a $k$-NN query in the same datasets.

Such results indicate Omni-histograms consistently delivered faster executions of $k$-NN queries, with and without orthogonal attributes, in comparison to the competitors. In particular, Equi-Width Omni-histogram and Compact-distance Omni-histogram outperformed the former members of the Omni-family, OmniSequential and Omni R-Tree by $37.4 \%$ and $32.3 \%$, on average, regarding all evaluated scenarios (datasets and values of $k$ ).

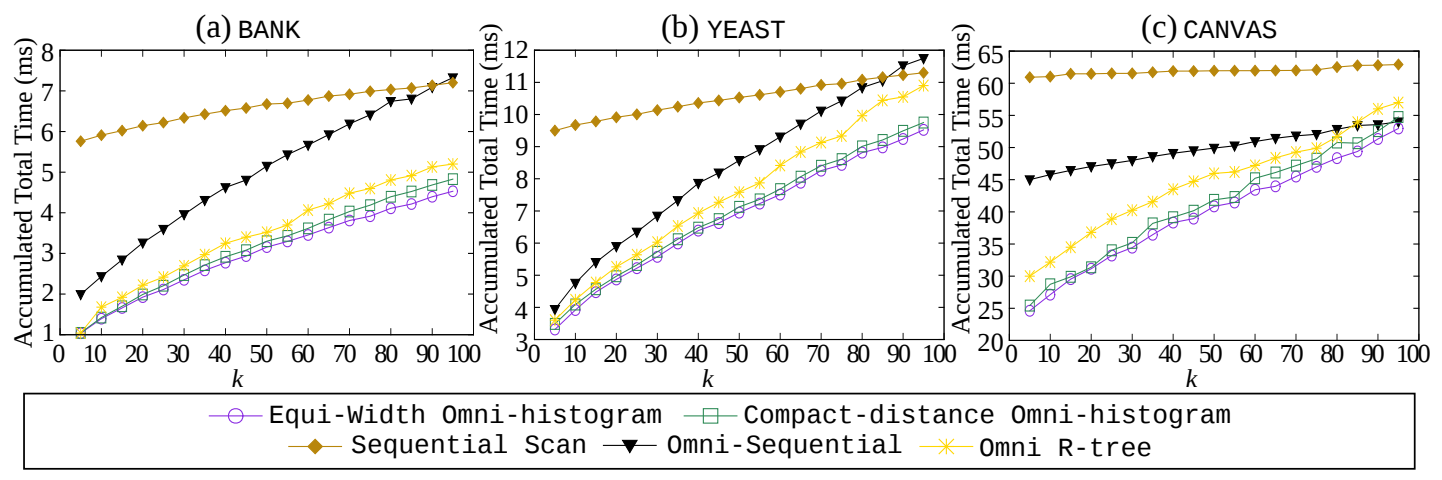

Figure 9: Comparison of Omni-histograms to Omni R-Tree, Omni-Sequential, and Sequential Scan regarding $k$-NN queries involving an orthogonal attribute. 


\section{CONCLUSION}

In this study, we extended the Omni-family by creating a new class of metric access methods, the Omni-histograms. Such methods distinguish themselves by their partition constraints, which split data elements into disjoint buckets by following an optimization function. The frequency within Omni-histogram regions is represented as either the number of elements or the data distribution of an orthogonal attribute. By using these stored frequencies, our approach enables the use of a bounded and incremental $k$-NN search, which limits the number of visited regions (bounding disk accesses) and reduces the number of distance calculations. Experiments on real datasets showed $k$-NN queries (with and without orthogonal attributes) are faster executed by Omni-histograms in comparison to previous Omni methods. In particular, Omni-histograms outperformed Omni-Sequential in up to $113 \%$ and Sequential Scan in up to $500 \%$. Future work includes the evaluation of other pivot selection strategies and algorithms in the setting of Omni-histograms.

Acknowledgments. The authors thank FAPERJ (G. E-26/010.101237/2018), CNPq, and CAPES for their financial support.

\section{REFERENCES}

[1] SILVA, Y. N. et al. Similarity queries: their conceptual evaluation, transformations, and processing. $V L D B$, Springer, v. 22, n. 3, p. 395-420, 2013. 2, 3

[2] YAO, B.; LI, F.; KUMAR, P. k-Nearest Neighbor queries and kNN-Joins in large relational databases (almost) for free. In: International Conference on Data Engineering. [S.l.]: IEEE, 2010. p. 4-15. 2, 3

[3] ZEZULA, P. Future trends in similarity searching. In: International Conference on Similarity Search and Applications. [S.1.]: Springer, 2012. p. 8-24. 2 ]

[4] ALY, A. M.; AREF, W. G.; OUZZANI, M. Spatial Queries with kNN and Relational Predicates. In: International Conference on Advances in Geographic Information Systems. [S.1.]: ACM, 2015. p. 28:1-28:10. 2

[5] WU, D.; CONG, G.; JENSEN, C. S. A framework for efficient spatial web object retrieval. The VLDB Journal, Springer, v. 21, n. 6, p. 797-822, 2012. 2

[6] CIACCIA, P.; PATELLA, M.; ZEZULA, P. M-tree: An efficient access method for similarity search in metric spaces. In: International Conference on Very Large Data Bases. [S.l.]: VLDB Endowment, 1997. p. 426-435. 2, 4

[7] SKOPAL, T.; POKORNỲ, J.; SNÁŠEL, V. Nearest neighbours search using the PM-tree. In: International Conference on Database Systems for Advanced Applications. [S.l.]: Springer, 2005. p. 803-815. 2

[8] TRAINA JR., C. et al. The Omni-family of all-purpose access methods: A simple and effective way to make similarity search more efficient. The VLDB Journal, Springer, v. 16, n. 4, p. 483-505, 2007. 2, 3, 4 
[9] CHÁvEZ, E. et al. Searching in metric spaces. ACM Computing Surveys, ACM, v. 33, n. 3, p. 273-321, 2001. 2, 3

[10] MOVSKO, J.; JAKUB, L.; SKOPAL, T. Clustered Pivot Tables for I/Ooptimized Similarity Search. In: International Conference on Similarity Search and Applications. [S.1.]: ACM, 2011. p. 17-24. 2, 3

[11] GRAEFE, G. Modern B-Tree Techniques. Foundations and Trends in Databases, Now Publishers, Inc., v. 3, n. 4, p. 203-402, 2011. 2

[12] GUTTMAN, A. R-trees: A Dynamic Index Structure for Spatial Searching. ACM Special Interest Group on Management of Data. 2

[13] BEDO, M. V. N. et al. Beyond Hit-or-Miss: A Comparative Study of Synopses for Similarity Searching. Journal of Information and Data Management, SBC, v. 9 , n. 1 , p. $36-51,2018.2$, 5

[14] TASAN, M.; OZSOYOGLU, Z. M. Improvements in distance-based indexing. In: Scientific and Statistical Database Management Conference. [S.1.]: IEEE, 2004. p. 161-170. 2, 3, 4, 5, 6

[15] VIEIRA, M. R. et al. Boosting knn queries by estimating suitable query radii. In: Scientific and Statistical Database Management Conference. [S.l.]: IEEE, 2007. p. 10. 2, 4, 6

[16] BUSTOS, C. et al. An empirical evaluation of intrinsic dimension estimators. In: International Conference on Similarity Search and Applications. [S.l.]: Springer, 2015. p. 125-137. 2

[17] PESTOV, V. Indexability, Concentration, and VC Theory. In: International Conference on Similarity Search and Applications. [S.l.]: ACM, 2010. p. 3-12. 2

[18] HETLAND, M. L. The basic principles of metric indexing. In: Swarm Intelligence for Multi-objective Problems in Data Mining. [S.l.]: Springer, 2009. p. 199-232. 3

[19] ZEZULA, P. et al. Similarity Search: The Metric Space Approach. [S.1.]: Springer, 2010. v. 1. 3.5

[20] BöHM, C.; KREBS, F. The k-Nearest Neighbour Join: Turbo Charging the KDD Process. KIS, Springer, v. 6, n. 6, p. 728-749, 2004. 3

[21] HJALTASON, G. R.; SAMET, H. Index-driven similarity search in metric spaces. ACM Transactions on Database Systems, ACM, v. 28, n. 4, p. 517-580, 2003. 4, 5

[22] IOANNIDIS, Y. The history of histograms. In: International Conference on Very Large Data Bases. [S.l.]: VLDB Endowment, 2003. p. 19-30. 5

[23] KÖNIG, A. C.; WEIKUM, G. A framework for the physical design problem for data synopses. In: International Conference on Extending Database Technology. [S.l.: s.n.], 2002. p. 627-645. 5 